Erwähnt werden möge noch, dass die Repulsivkraft, streng genommen, kaum als constant anzunehmen ist. Denn da Wärme- und Lichtintensität der Sonne periodischen Schwankungen unterworfen sind, muss, unter Berlicksichtigung der Constanz der Gesammtenergie, auch die elektrische

Berlin, 1897 December.
Intensität varïren, wodurch eine ganz exacte Bestimmung derselben, streng genommen, unmöglich wird. Indess werden derartige relativ geringe Schwankungen durch Beobachtungen nicht nachweisbar sein und ausserhalb der Grenzen der Wahrnehmbarkeit liegen.

\title{
Zur Theorie der intermediären Bahnen des Mondes.
}

Von A. W. Krassnow, Privatdocent der K. Universität Kasan.

Indem wir, der Einfachheit wegen, die Neigung der Mondbahn gegen die Ekliptik vernachlässigen, lässt sich die geocentrische Mondbewegung aus der Hamilton-Jacobi'schen Gleichung bestimmen:

$$
\frac{\mathrm{d} S}{\mathrm{~d} t}+1 / 2\left[\left(\frac{\mathrm{d} S}{\mathrm{~d} r}\right)^{2}+\frac{\mathrm{I}}{r^{2}}\left(\frac{\mathrm{d} S}{\mathrm{~d} \lambda}\right)^{2}\right]=\frac{\mathrm{I}}{r}+1 / 4 \alpha^{2} r^{2}(\mathrm{I}+3 \cos 2 \omega)+\beta r^{3}(3 \cos \omega+5 \cos 3 \omega),
$$

wo $\infty$ den Längenunterschied des Mondes und der Sonne bedeutet und die anderen Bezeichnungen einen leicht zu verstehenden Sinn haben.

Nimmt man an, dass die geocentrische Sonnenbewegung in einem Kreise mit einer constanten Winkelgeschwindigkeit

$n$ vor sich geht, so ist $\lambda=\infty+n t+$ const. Führt man anstatt $\lambda$ die neue Variable $\boldsymbol{\omega}$ ein, so $\operatorname{sind} \frac{\mathrm{d} S}{\mathrm{~d} \lambda}$ und $\frac{\mathrm{d} S}{\mathrm{~d} t}$ resp. durch $\frac{\mathrm{d} S}{\mathrm{~d} \omega}$ und $\frac{\mathrm{d} S}{\mathrm{~d} t}-n \frac{\mathrm{d} S}{\mathrm{~d} \omega}$ zu ersetzen. Damit wird

$$
\frac{\mathrm{d} S}{\mathrm{~d} t}+1 / 2\left[\left(\frac{\mathrm{d} S}{\mathrm{~d} r}\right)^{2}+\frac{1}{r^{2}}\left(\frac{\mathrm{d} S}{\mathrm{~d} \omega}\right)^{2}\right]-n \frac{\mathrm{d} S}{\mathrm{~d} \omega}=\frac{1}{r}+1 / 4 \alpha^{2} r^{2}(1+3 \cos 2 \omega)+\beta r^{3}(3 \cos \omega+5 \cos 3 \omega),
$$

also ergiebt sich unmittelbar das Integral der lebendigen Kraft (Jacobi's Integral) :

$$
\left(\frac{\mathrm{d} \Sigma}{\mathrm{d} r}\right)^{2}+\frac{\mathrm{I}}{r^{2}}\left(\frac{\mathrm{d} \Sigma}{\mathrm{d} \omega}\right)^{2}-2 n \frac{\mathrm{d} \Sigma}{\mathrm{d} \omega}=\frac{2}{r}-\frac{\mathrm{I}}{a}+1 / 2 \alpha^{2} r^{2}(\mathrm{I}+3 \cos 2 \omega)+2 \beta r^{3}(3 \cos \omega+5 \cos 3 \omega),
$$
wenn $S=\frac{t}{2 a}+\Sigma$ und $\Sigma$ von $t$ unabhängig ist. Nun ist das Product $n \frac{\mathrm{d} \Sigma}{\mathrm{d} \omega}$ sehr nahe eine Constante. Setzt man wie gewöhnlich $u=\frac{\mathrm{r}}{r}$ und bemerkt ausserdem, dass das

$$
\Sigma=\int \frac{\mathrm{d} u}{u} \sqrt{\frac{2}{u}-\frac{b}{u^{2}}-p+1 / 2 \frac{\alpha^{2}}{u^{4}}}+\int \mathrm{d} \omega \sqrt{p+\frac{6 \beta}{c^{5}} \cos \omega+3 / 2 \frac{\alpha^{2}}{c^{4}} \cos 2 \omega},
$$

wo $b=\frac{1}{a}+2 n \sqrt{p},-c$ als eine im Voraus gegebene Constante der Aufgabe anzusehen ist und $a$ und $p$ zwei Integrationsconstanten bilden.

Somit haben wir für unsere intermediäre Bahn

$$
\begin{gathered}
A=-\frac{t}{2 a^{2}}+\frac{\mathrm{I}}{2 a^{2}} \int \frac{\mathrm{d} u}{u \sqrt{2 u^{3}-b u^{2}-p u^{4}+1 / 2 \alpha^{2}}} \\
B=\frac{1}{2} \int \frac{\mathrm{d} \omega}{\sqrt{p+\frac{6}{c^{5}} \cos \infty+3 / \frac{\alpha^{2}}{c^{4}} \cos 2 \infty}}-\frac{1}{2} \int \frac{\left.n+\frac{n}{\sqrt{p}} \frac{\mathrm{I}}{u}\right) \mathrm{d} u}{\sqrt{2 u^{3}-b u^{2}-p u^{4}+1 / 2 \alpha^{2}}} \\
b=\frac{1}{a}+2 n \sqrt{p} ; \quad \lambda=\infty+n t+\text { const. }
\end{gathered}
$$

Diese Auflösung enthält drei elliptische Integrale und ist bis zur zweiten Ordnung inclusive genau. Die numerischen Werthe der Coefficienten $1 / 2 \alpha^{2}, 3 / 2 \frac{\alpha^{2}}{c^{4}}$ und $\frac{6 \beta}{c^{5}}$ könn. ten wir unbestimmt bleiben lassen.
Die oben angeführten Bemerkungen sind durch die Abhandlung des Herrn Dr. G. W. Hill im Astronomical Journal Nr. 4 I9 veranlasst.

Wie man verfahren kann, wenn man vermuthet, dass die Sonne keine kreisförmige, sondern eine beliebige Bewegung hat, 
darüber siehe das 3. Capitel meiner Inaugural-Magisterschrift $\mid$ schnell die Glieder mit $\alpha^{2}$ und $\alpha^{4}$ der Säcularbeschleunigung *Theorie der Sonnenungleichheiten der Mondbewegung \&, der Mondbewegung, wie es daselbst im 8. Capitel auseinKasan 1895 (in russischer Sprache). Die Methode giebt sehr andergesetzt ist.

Kasan, Sternwarte, 1897 Dec. 25.

A. W. Krassnow.

\section{Minor Planet Observations at Windsor, N. S. Wales.}

\section{By Fohn Tebbutt.}

The accompanying observations have been made with | are headed as "Geocentric *. The headings should, however, the 8 inch equatorial refractor and filar-micrometer in a be »Geocentric Apparent \& as no correction was applied for bright field. I notice that the planet-coordinates published aberration. I deem it advisable to offer this explanation in by $m e$ in No. 2842,2961 and 3020 of the Astr. Nachr. (order to avoid doubt in the use of the data.*)

\begin{tabular}{l|l|l|l|l|l|l|l|l|l|l|}
\hline 1897 & Winds. M.T. & $\Delta$ RA. & $\Delta$ NPD. & Cp. & RA. app. & $\log p . \Delta$ & NPD. app. & $\log p . \Delta$ & Red. ad 1. app. & $*$ \\
\hline
\end{tabular}

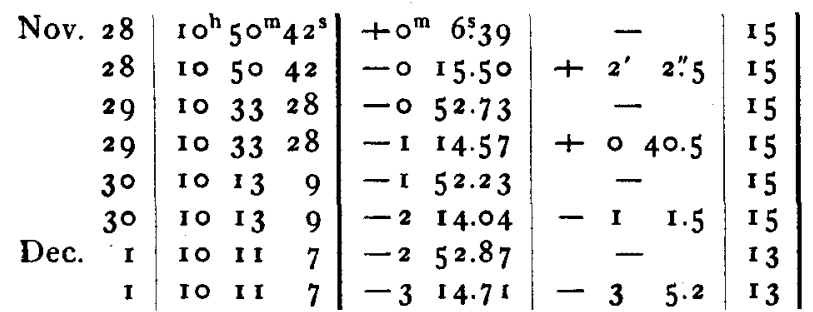

(6) Hebe.

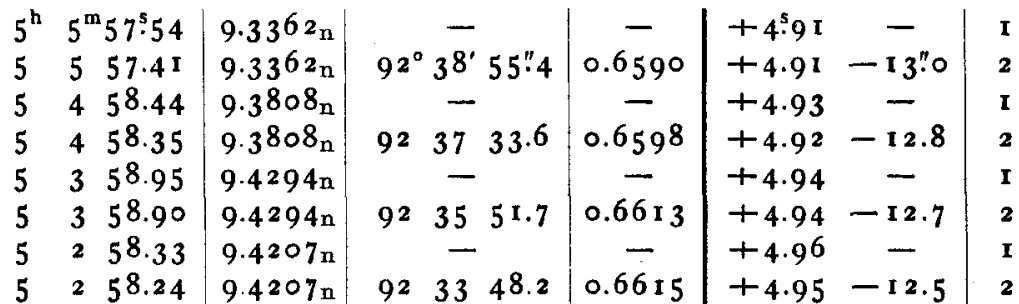

(I 1) Parthenope.

\begin{tabular}{|c|c|c|c|c|c|c|c|c|c|}
\hline \multicolumn{2}{|c|}{ Sept. 26} & 9 & $3^{6}$ & 54 & +0 & $29.4^{6}$ & +6 & I 4.5 & 20 \\
\hline & 29 & 10 & 34 & 31 & +0 & 14.23 & -4 & 8.1 & ro \\
\hline & 29 & IO & 34 & 31 & -0 & $3^{8.04}$ & - & & 10 \\
\hline & 30 & 10 & 44 & 4 & -0 & $3^{6.90}$ & +2 & 39.9 & 3 \\
\hline & 30 & 10 & 44 & 4 & $-I$ & 28.92 & - & & 3 \\
\hline Oct. & I & Io & $3^{6}$ & $5^{8}$ & +1 & 31.37 & -4 & 27.1 & 10 \\
\hline & 6 & 10 & 20 & 49 & +2 & $35.7 \mathrm{I}$ & +9 & 41.8 & IO \\
\hline & 7 & 10 & 24 & 22 & +1 & 42.56 & +15 & 57.8 & 7 \\
\hline & 7 & 10 & 24 & 22 & -6 & 41.88 & +2 & 27.0 & 7 \\
\hline & 8 & Io & 9 & 7 & -3 & $5^{8.24}$ & - & & 6 \\
\hline & 8 & 10 & 9 & 7 & -7 & 34.31 & +8 & 33.0 & 6 \\
\hline & I I & IO & $1 \mathrm{I}$ & 3 & +3 & 55.78 & - I 5 & 0.2 & 7 \\
\hline & I I & IO & I I & 3 & $+I$ & $10.6 \mathrm{I}$ & -5 & $5 \cdot 5$ & 7 \\
\hline & I 2 & 9 & 50 & I I & +3 & $4 \cdot 34$ & -9 & 25.6 & Io \\
\hline & 12 & 9 & 50 & II & +0 & I 9.18 & +0 & 28.9 & IO \\
\hline & 14 & I I & 18 & 59 & +4 & 7.85 & - & $4^{6.4}$ & IO \\
\hline & 14 & I I & 18 & 59 & +1 & $17.6 \mathrm{I}$ & + & 47.9 & IO \\
\hline & 15 & 9 & 34 & I I & +3 & 21.00 & + & 3.6 & 10 \\
\hline & 15 & 9 & 34 & I I & +0 & 30.64 & & - & 10 \\
\hline & I 6 & 8 & $3^{8}$ & $2 \mathrm{I}$ & +2 & 32.54 & +4 & 55.5 & 12 \\
\hline & I 6 & 8 & $3^{8}$ & 21 & -0 & 17.79 & & & 12 \\
\hline & 17 & 9 & $5^{2}$ & I 2 & $+I$ & 40.13 & +10 & 3.9 & 10 \\
\hline & I 7 & 9 & $5^{2}$ & I 2 & $-I$ & 10.13 & & & 10 \\
\hline
\end{tabular}

\begin{tabular}{|c|c|c|c|}
\hline I & 12 & 24.00 & $9.5653 \mathrm{n}$ \\
\hline I & 9 & 53.93 & $9.395^{\circ} \mathrm{n}$ \\
\hline I & 9 & 53.22 & $9.395^{\circ} \mathrm{n}$ \\
\hline $\mathbf{I}$ & 9 & 2.81 & $9 \cdot 3449 n$ \\
\hline I & 9 & 2.35 & $9.3449 n$ \\
\hline I & 8 & I 1.28 & $9.3534 n$ \\
\hline I & 3 & 49.33 & $9.3^{2} 33 n$ \\
\hline $\mathbf{I}$ & 2 & 56.19 & $9.2880_{\mathrm{n}}$ \\
\hline I & 2 & $5^{6.05}$ & $9.288 \circ_{n}$ \\
\hline $\mathbf{I}$ & 2 & 3.92 & $9.3315 n$ \\
\hline $\mathbf{I}$ & 2 & $3^{6} 3$ & 9.33 I $5 n$ \\
\hline o & 59 & 25.35 & $9.2610_{n}$ \\
\hline 0 & 59 & 25.15 & $9.26 \mathrm{I} O_{\mathrm{n}}$ \\
\hline$\circ$ & 58 & 33.92 & $9.33^{\circ} 6_{n}$ \\
\hline o & $5^{8}$ & 33.72 & $9.33 \circ 6_{n}$ \\
\hline 0 & $5^{6}$ & 46.92 & $7.9^{82} \mathrm{O}_{\mathrm{n}}$ \\
\hline 0 & $5^{6}$ & $47.2 \circ$ & $7.9^{8} 2 \mathrm{O}_{\mathrm{n}}$ \\
\hline 0 & $5^{6}$ & 0.07 & $9 \cdot 337 \mathrm{I}_{\mathrm{n}}$ \\
\hline 0 & $5^{6}$ & 0.23 & $9.3371_{n}$ \\
\hline 0 & 55 & 11.62 & $9.49 \times 3 \mathrm{n}$ \\
\hline 0 & 55 & $11.8 \mathrm{I}$ & $9.49 \times 3 n$ \\
\hline 0 & 54 & $19.2 \mathrm{I}$ & $9.2 I I I n$ \\
\hline 0 & 54 & 19.47 & $9.211 I_{n}$ \\
\hline
\end{tabular}

\begin{tabular}{ccc|c|cc|c}
90 & 20 & 20.7 & 0.6851 & +4.33 & -29.2 & 3 \\
90 & 41 & 1.5 & 0.6808 & +4.37 & -29.3 & 4 \\
& - & & - & +4.37 & - & 5 \\
90 & 47 & 49.4 & 0.6792 & +4.38 & -29.4 & 4 \\
& - & & - & +4.38 & - & 5 \\
90 & 54 & 35.8 & 0.6782 & +4.39 & -29.4 & 6 \\
91 & 27 & 11.0 & 0.6722 & +4.44 & -29.4 & 7 \\
91 & 33 & 27.0 & 0.6708 & +4.45 & -29.4 & 7 \\
91 & 33 & 26.0 & 0.6708 & +4.45 & -29.4 & 8 \\
& - & & - & +4.46 & - & 9 \\
91 & 39 & 32.0 & 0.6699 & +4.46 & -29.4 & 8 \\
91 & 57 & 16.4 & 0.6660 & +4.47 & -29.3 & 10 \\
91 & 57 & 14.4 & 0.6660 & +4.48 & -29.3 & 11 \\
92 & 2 & 51.0 & 0.6656 & +4.48 & -29.3 & 10 \\
92 & 2 & 48.8 & 0.6656 & +4.48 & -29.3 & 11 \\
92 & 14 & 5.6 & 0.6608 & +4.49 & -29.2 & 12 \\
92 & 14 & 4.6 & 0.6608 & +4.49 & -29.2 & 10 \\
92 & 18 & 55.6 & 0.6628 & +4.49 & -29.2 & 12 \\
92 & -23 & 47.6 & 0.6652 & +4.50 & -29.1 & 12 \\
92 & -12 & & - & +4.50 & - & 10 \\
92 & 28 & 56.0 & 0.6594 & +4.50 & -29.1 & 12 \\
& - & & - & +4.50 & - & 10 \\
& & & & & & 10
\end{tabular}

(194) Prokne.

\begin{tabular}{|c|c|c|c|c|c|c|c|c|c|c|c|c|c|c|c|c|c|c|c|}
\hline $\begin{array}{r}\text { Aug. } \\
\text { I } 5 \\
\\
15 \\
\text { I } 6\end{array}$ & $\begin{array}{r}9 \\
10\end{array}$ & $\begin{array}{ll}44 & 55 \\
44 & 55 \\
44 & 56\end{array}$ & $\begin{array}{l}-3 \\
-3 \\
+7\end{array}$ & $\begin{array}{l}43.67 \\
45.48 \\
25.17\end{array}$ & $\begin{array}{l}+ \\
-\end{array}$ & 5 & 53.5 & $\begin{array}{l}15 \\
15 \\
10\end{array}$ & $\begin{array}{l}22 \\
22 \\
22\end{array}$ & $\begin{array}{l}33 \\
33 \\
33\end{array}$ & $\begin{array}{l}56.95 \\
56.91 \\
28.27\end{array}$ & $\begin{array}{l}9.5643 \mathrm{n} \\
9.5643 \mathrm{n} \\
9.4164 \mathrm{n}\end{array}$ & 93 & $\frac{2}{20}$ & 28.7 & $\begin{array}{c}0.663^{1} \\
-\overline{6}_{528}\end{array}$ & $\begin{array}{l}+4.10 \\
+4.10 \\
+4.14\end{array}$ & $\begin{array}{c}-22.2 \\
- \\
-21.7\end{array}$ & $\begin{array}{l}13 \\
14 \\
15\end{array}$ \\
\hline
\end{tabular}
$\left.{ }^{*}\right)$
haben wird. $K r$. 